\title{
INTERNET: UNA HERRAMIENTA PARA LA DESLOCALIZACIÓN DE LA ENSEÑANZA DEL DERECHO. ANÁLISIS DE UNA EXPERIENCIA*
}

\author{
Roberto Navarro Dolmestch ${ }^{* *}$ y Juan José Valenzuela Silva ${ }^{* * *}$ \\ "Nadie pondrá en duda que el legislador debe poner el mayor empeño \\ en la educación de los jóvenes. En las ciudades donde no ocurre asi, ha \\ resultado en detrimento de la estructura política, porque la educación \\ debe adaptarse a las diversas constituciones, ya que el carácter \\ peculiar de cada una es lo que suele preservarla, del mismo modo que \\ la estableció en su origen: el espíritu democrático, por ejemplo, la \\ democracia, y el oligárquico, la oligarquía: y el espiritu mejor, en fin, \\ es causa de la mejor constitución".
}

ARISTÓteles, La Política, VIII.1.

El aprendizaje del Derecho es un proceso complejo que posee determinados rasgos característicos que lo identifican respecto del aprendizaje, desde luego, de las ciencias exactas y, en menor medida, del de las demás ciencias sociales.

Este trabajo pretende dar cuenta de la experiencia concreta de aplicación de las modernas tecnologías de la información basadas en la red Internet, a la enseñanza de contenidos de ciertas áreas del Derecho público. Para ello, partiremos intentando establecer las principales características que definen al actual modelo de enseñanza del Derecho, para luego exponer sobre el tema central de esta ponencia: la utilización de la Internet como un herramienta de deslocalización del proceso de enseñanza.

De todas formas, es menester partir con una primera advertencia, en el sentido que la experiencia de este trabajo está actualmente desarrollándose en la Escuela de Derecho de la Universidad de Talca, por lo que no es posible aún contar con resultados definitivos y, por tanto, con evaluaciones globales de la técnica empleada.

\section{DEFINICIÓN DEL MODELO TRADICIONAL DE ENSEÑANZA DEL DERECHO.}

Tratar de establecer las características principales del actual modelo es una tarea que no puede estar exenta de puntos de discordia, más aún cuando éste es el que

\footnotetext{
'Patrocinio: Jean Pierre Matus Acuña, Abogado PU.C de Chile, Dr y Mg en Derecho por la Universidad Autónoma de Barcelona. Profesor Asistente de Derecho penal en la Universidad de Talca.

"Egresado de Derecho. Ayudante del Departamento de Derecho Público y Procesal, Universidad de Talca.

"**Estudiante de Derecho. Ayudante de Derecho procesal III, Universidad de Talca.
} 
se aplica en la casi totalidad de las asignaturas de Derecho, al menos en la Facultad de Ciencias Jurídicas Sociales ${ }^{1}-{ }^{2}$.

\subsection{Perspectiva subjetiva: Estructura dual.}

El modelo tradicional de enseñanza del Derecho se caracteriza, en primer lugar desde una perspectiva subjetiva, por la existencia de dos sujetos claramente delimitados que participan en el proceso de enseñanza: el profesor y los alumnos. Esta estructura dual excluye la participación de otros sujetos, tales como la comunidad, otros operadores jurídicos (jueces, abogados) o personas específicas que acuden a órganos jurisdiccionales o requieren orientación jurídica.

Los alumnos, a pesar de su pluralidad física, sólo constituyen un sujeto del proceso debido -a nuestro juicio- a dos causas. La primera, en conexión con la perspectiva objetiva que se analizará más adelante, ya que todos los alumnos, a pesar de su pluralidad y singularidad deben adoptar el único rol que el modelo les permite asumir $^{3}$. Y la segunda razón tiene un carácter económico y de definición del acceso a la educación, representada por el excesivo número de alumnos que componen cada curso ${ }^{4}$ que impide la atención personalizada.

1.2. Perspectiva objetiva: La definición de los roles; Sujeto activo y sujeto pasivo.

En segundo lugar, desde una perspectiva objetiva, esta estructura dual tiene como consecuencia que ambos sujetos adoptan un rol determinado e inamovible en todo el proceso. Mientras que el profesor es el sujeto activo del proceso, los alumnos constituyen el centro pasivo de éste. En función de estos roles, el profesor es el que debe entregar el conocimiento que sólo él posee y los alumnos recibirlo, lo que significa que la estructura dual con roles determinados se construye sobre la base de relaciones unidireccionales 'profesor-alumno', eliminándose las posibilidades de retroalimentación directa.

\footnotetext{
${ }^{1}$ Aunque no podemos contar con datos precisos, esta afirmación se basa en la primera forma de conocimiento: la experiencia personal.

${ }^{2}$ Esta tarea será asumida desde diferentes perspectivas; a saber: a) Perspectiva subjetiva; $b$ ) Perspectiva objetiva; c) Distribución de los tiempos; d) Localización y temporalización; y e) Evaluación.

${ }^{3}$ Ver infra, §1-1..2

${ }^{4}$ El número de alumnos regulares en la carrera de Derecho en la Universidad de Talca se ha ido incrementando paulatinamente en los últimos cinco años a una tasa promedio anual de $11,9 \%$. Si tomamos como supuesto que en cada nivel hay dos secciones de las asignaturas (10 secciones en total, por cinco niveles), el promedio de alumnos por cada sección (curso) en los últimos cinco años ha sido de 65 personas.
} 
Encontrar la razón que explique esta perspectiva del modelo no es una tarea fácil. Lo que es claro es que esta perspectiva objetiva del modelo tradicional de enseñanza del Derecho, tiene una connotación política definida en el sentido que éste no es un modelo democrático, aunque esto no debe entenderse como afirmación de la extrapolación de un concreto sistema político a la enseñanza, ya que no poseemos elementos suficientes que nos permitan hacer tal afirmación ${ }^{5}$. Tan sólo queremos establecer que la estructura de las relaciones entre los sujetos en el modelo tradicional, no es propiamente democrática.

\subsection{Distribución de los tiempos.}

Una tercera perspectiva desde la cual se puede caracterizar el modelo tradicional de enseñanza, está determinada por la distribución de los tiempos. En efecto, al partir este modelo de la figura de un profesor con un rol súper activo y del alumno-receptor. debe concluirse en la necesidad de aumentar los tiempos destinados a las sesiones en las cuales se desarrolla el proceso de enseñanza, por cuanto a mayor cantidad de tiempo en que los alumnos estén en contacto unidireccional con el profesor, mayor es la cantidad de contenidos que podrán ser abarcados. En este modelo, la cantidad de tiempo que se destina a la clase presencial se asocia a una mejor calidad de la educación. La experiencia de la Facultad de Ciencias Jurídicas de la Universidad de Talca, confirma esta afirmación, ya que en promedio, los alumnos de la carrera de Derecho deben dedicar 42.9 horas cronológicas a la semana, según la distribución de creditaje asignado en el plan curricular ${ }^{6}$. Este aspecto también presenta una connotación política, porque esta distribución y exigencia temporales a los estudiantes es ideal en sistemas políticos autoritarios, ya que mantener a los alumnos ocupados en 'actividades académicas' los desvincula de otras actividades necesarias, como las relativas a la organización estudiantil.

\footnotetext{
${ }^{5}$ Asumir la labor de encontrar los elementos que permitan afirmar dicha relación escapa al objeto de esta ponencia.

${ }^{6}$ Si se considera que un 'crédito' ( 45 minutos) es el tiempo que cada alumno debe dedicar semanalmente a cada asignatura y que, "Para efectos de cálculo, el total de los créditos anuales se considerarán con el doble de créditos que los semestrales" (art. 44 Reglamento de Régimen de Estudios de la Universidad de Talca). La distribución por nivel se aprecia en el siguiente cuadro (Distribución de la carga académica por año académico, según creditaje (en créditos y horas cronológicas):
}

\begin{tabular}{|l|l|l|l|l|l|}
\cline { 2 - 6 } \multicolumn{1}{c|}{} & Primer año & Segundo año & Tercer año & Cuarto año & Quinto año \\
\hline $\begin{array}{c}\text { Total global. Asignaturas anuales y } \\
\text { semestrales (créditos) }\end{array}$ & 62 & 60 & 72 & 53 & 39 \\
\hline Total en horas cronológicas & 46,5 & 45.0 & 54.0 & 39.8 & 29.3 \\
\hline
\end{tabular}


1.4. La ubicación espacial del proceso: La localización y temporalización.

La cuarta perspectiva de análisis es consecuencial de las tres anteriores en el sentido que el modelo tradicional, requiere para su concreción, de un lugar físico determinado -generalmente la sala de clases-.

Esta característica implica una necesidad de temporalización, ya que profesor y alumnos deben reunirse en un lugar y a una hora determinada.

\subsection{La evaluación.}

Como un modelo sobre el proceso de enseñanza del Derecho, que es el que intentamos caracterizar, éste requiere de un sistema de evaluación. De acuerdo al rol pasivo que al alumno le corresponde desempeñar en el modelo tradicional, la evaluación debe reflejar la cantidad que éste logró retener; la evaluación se presenta como una herramienta que tiene por objeto medir el nivel de recepción y retención de los contenidos que el profesor ha entregado durante un período previo.

\subsection{A modo de recapitulación.}

Con los elementos anteriormente expuestos, estamos en condiciones de afirmar, que el modelo tradicional de enseñanza del Derecho es aquél en el cual el proceso de aprendizaje se concibe altamente localizado y temporalizado, unidireccionalmente en una relación que asocia al binomio 'profesor-alumno' en relación de roles de sujeto activo a receptor pasivo de los conocimientos, cuyo monopolio ostenta el profesor y cuya única herramienta de evaluación mide el nivel de recepción y retención de conocimientos.

\section{EL MODELO TRADICIONAL: VISIÓN VALORATIVA.}

La visión de conjunto que hemos esbozado anteriormente ciertamente es limitada, ya que da cuenta del modelo tradicional, pero sólo desde una perspectiva descriptiva. Es decir, esta definición -en base a los elementos anteriores- no entrega ninguna información sobre lo deseable que es el modelo o las deficiencias estructurales que pueda poseer. Sin embargo, tampoco estamos en condiciones de ofrecer una respuesta empírica generalizadora que responda las preguntas que implica esta visión valorativa ${ }^{7}$, sino que sólo estamos en condiciones de establecer determinadas pautas

\footnotetext{
${ }^{7}$ La complejidad del proceso de enseñanza del Derecho hace extremadamente dificultosa la tarea de evaluar modelos en función de sus resultados. Ello se sale de los límites impuestos por el objetivo de este trabajo y escapa a nuestras competencias y conocimientos.
} 
que permitan dar luz sobre la deseabilidad del sistema, siempre teniendo presente que tales definiciones serán, principalmente, de carácter teórico.

\subsection{Visión valorativa (I): El modelo tradicional como modelo autoritario.}

Los elementos que conforman el modelo tradicional de enseñanza del Derecho permiten sostener que éste es de corte autoritario, porque parte de la premisa que la única fuente válida de conocimiento es el profesor y como tal él podrá dirigir a los alumnos hacia otras fuentes específicas que él considere, lo que se traducirá en una 'bibliografía complementaria' que acompañará al programa de la asignatura; el profesor actúa como una especie de 'filtro' de fuentes señalando cuáles son válidas y cuáles no. Ello se ve profundizado por la relación unidireccional con que se caracterizó al modelo, unido al rol pasivo que deben asumir los alumnos, lo que supone la implementación de relaciones de jerarquía.

\subsection{Visión valorativa (II): El modelo tradicional no es eficiente.}

Las relaciones que admite dentro de sí el modelo tradicional, no permite el desarrollo eficiente de las potencialidades de los estudiantes, en el sentido que éste se basa en una entrega de conocimientos que los alumnos deben retener, sin que el sistema espere de ellos una respuesta crítica frente a los datos que el profesor ha entregado. Por eso, dentro del modelo tradicional, la evaluación asume el rol antes expuesto ${ }^{8}$ que pasa a ser un control de cantidad más que de calidad.

Para lograr los objetivos, en el modelo tradicional deben emplearse grandes cantidades de tiempo (localización y temporalización), lo que hace que los alumnos permanezcan ocupados en clases y luego absorbiendo los contenidos entregados por el profesor $^{9}$, sin tener oportunidad real de profundizar o dedicar tiempo a la investigación de pregrado o de participar en otras actividades también necesarias.

\section{LA RED INTERNET COMO UN SOPORTE VIABLE DEL PROCESO DE ENSEÑANZA.}

El desarrollo y la relativa masificación que la red Internet ha tenido en el último tiempo, plantea la interrogante de si puede ésta ser utilizada en la enseñanza del Derecho y el tema que convoca este encuentro es una prueba de ello. Creemos que no existen limitaciones tecnológicas que impidan incorporar a la informática como una herramienta válida dentro del proceso educativo. Esto es una realidad que cada día

\footnotetext{
${ }^{8}$ Ver supra, $\$ 1-1.5$.

${ }^{9}$ Ver supra, §1-1.3.
} 
recobra mayor relevancia. Lo importante es definir el rol que ésta cumplirá y no perder la perspectiva que ésta sólo es una herramienta que coadyuva al proceso.

\section{UN PROBLEMA DE DEFINICIÓN PREVIA: ¿LA INFORMÁTICA COMO HERRAMIENTA ANEXA O COMO HERRAMIENTA CENTRAL (SOPORTE) DEL PROCESO?}

Desde luego, Informática y Derecho tienen una serie de puntos de yuxtaposición que cada día van en aumento. Por ello, expresiones tales como 'informática jurídica documental', 'informática jurídica decisional'10, 'derecho informático ${ }^{11}$ no son sino expresiones de un mismo fenómeno: La penetración irreversible de la informática y de las tecnologías de la información en todos los campos y en todas las actividades.

Una vez que se ha contestado afirmativamente, la interrogante respecto de la factibilidad de la aplicación de la informática como herramienta pedagógica -algo así como una 'informática jurídica pedagógica- debe decidirse sobre el rol real que a ésta se le asignará. $Y$ en este aspecto, la informática jurídica pedagógica, puede utilizarse como una herramienta anexa a través del empleo, por ejemplo, de bases de datos que condensen e interrelacionen grandes volúmenes de información (jurisprudencia, repertorio de legislaciones, etcétera) o de la automatización de ciertas funciones (formatos prediseñados de escritos, v.gr.) o, por el contrario, puede asignársele a esta informática jurídica pedagógica un rol mucho más activo como soporte -no exclusivodel proceso de enseñanza.

Estas decisiones estarán muchas veces condicionadas por factores externos, como la disponibilidad de servicio adecuados al interior de la facultades o el nivel de acceso que los alumnos tengan a la específica tecnología de que se trata. A ello deben sumarse criterios económicos de decisión, como la disponibilidad de recursos económicos y personal capacitado para ejecutar las funciones necesarias.

Y ésta es precisamente la decisión, que en forma experimental, se ha tomado en el área de Derecho penal y procesal penal de la Facultad de Ciencias Jurídicas de la Universidad de Talca.

\footnotetext{
${ }^{10}$ Sobre ésta y la anterior, ver BAUZÁ REILLY, Marcelo, "Informática jurídica en una Facultad de Derecho. Roles y perspectivas", en Revista Electrónica de Derecho Informático, http://publicaciones.derecho.org/redi/ $\mathrm{N}^{\circ} .06$ __Enero_de_1999/bauza (25-09-2000).

${ }^{11} \mathrm{Al}$ respecto, PEÑARANDA. Héctor, "La informática jurídica y el derecho informático como ciencias. El derecho informático como rama autónoma del Derecho", en Revista Electrónica de Derecho Informático http://publicaciones.derecho.org/redi/No_03___Octubre_de_1998/hector (25-09-2000).
} 
Es así como este año se organizaron dos proyectos en los cuales la red Internet tuvo un rol fundamental. El primero de ellos es el Diplomado en Litigación Oral y Nuevo Proceso Penal y el segundo, la Tutoría de Derecho procesal III (recursos) la que actualmente está en pleno desarrollo.

Ambos proyectos son muy ambiciosos, pero con un gran esfuerzo del Profesor guía y de los ayudantes, se pudo llevar a cabo.

En cuanto a la asignatura que aún se está impartiendo, que es el de Derecho procesal III, comenzó como una propuesta de la Facultad -no institucional- para que aquéllos alumnos que habían reprobado dicha asignatura durante el primer semestre, la pudieran cursar durante el segundo semestre ${ }^{12}$.

Esto nos llevó a esforzarnos al máximo en pos de este gran desafío, que era, en conjunto con el profesor, crear el primer curso enteramente por Internet en la Universidad de Talca. Para llevar a cabo este proyecto se diseñó una página web del curso(http://www.utalca.cl/Facultad/derecho/diplomado/tutoria/index.html),

organizar un organigrama con todos los puntos a tocar en las distintas sesiones (syllabus), la bibliografía a utilizar, el sistema de evaluaciones y en fin, todo aquello que fuese necesario para darle a los alumnos un cabal conocimiento de la materia a tratar en el curso.

Hasta el momento, la asignatura ha funcionado correctamente, a través de la modalidad de trabajos semanales dados por el Profesor en la página web del curso y, además, todas las consultas pertinentes, respecto a la cátedra, se hacen vía e-mail a los ayudantes o al Profesor.

Este trabajo no ha estado exento de dificultades. A pesar de la cobertura de computadores en el Campus Lircay ${ }^{13}$, estas cifras no dan cuenta de problemas como la caída del servidor en el que está alojado el sitio y, por tanto, la imposibilidad de acceso a él, como ya ha ocurrido durante la tercera semana de septiembre.

También debemos destacar que este es un proyecto innovador en nuestra Universidad, por lo que hemos debido ceder en algunos puntos, como por ejemplo, las ayudantías siguen siendo presenciales y muchos casos se han debido explicar no por correo, sino que en explicaciones personales del Profesor.

\footnotetext{
${ }^{12}$ La asignatura de Derecho procesal III del semestre primavera (segundo de este año) se está dictando por tutoría, lo que significa que es para alumnos que reprobaron tal asignatura durante el semestre anterior. este curso ofrecía oportunidades de implementación del proyecto, por cuanto en éste sólo hay ocho alumnos inscritos.

${ }^{13}$ Ver infra, $§ 6.2$. , nota al pie número 15 .
} 
En último lugar, debemos dar a conocer que ya se han efectuado evaluaciones a los alumnos, siendo la calificación promedio del curso de 5,1, ya que se nota el entusiasmo por la innovación y los modernos elementos incorporados a la vía académica cotidiana de los alumnos.

\section{HACIA UN NUEVO MODELO DE ENSEÑANZA: EL MODELO DESLOCALIZADO COMO SUPERACIÓN DEL MODELO TRADICIONAL. LA EXCLUSIÓN DE UN MERO CAMBIO DE SOPORTE.}

La utilización de la red Internet como soporte no exclusivo del proceso de enseñanza del Derecho permite la superación o, al menos, corrección, de muchos de los elementos característicos del modelo tradicional.

En efecto, abre las puertas para la incorporación de más y variados sujetos al proceso, lo que permite la superación de la estructura dual.

Respecto de la definición de los roles, un modelo deslocalizado con la ayuda de las tecnologías de la información aumenta las posibilidades reales de romper el paradigma tradicional 'profesor-sujeto activo/alumno--sujeto pasivo', aunque, claro está, no es posible establecer una relación de identidad entre la adopción de un modelo deslocalizado y tal superación.

Si bien es cierto, la adopción de un modelo deslocalizado no significa disminuir la asignación temporal que es propia del modelo tradicional, no es menos cierto que el nuevo modelo, permite una mejor y más eficiente distribución del tiempo en función de las necesidades personales de cada estudiante.

Por último, este modelo deslocalizado precisa de un nuevo instrumento de evaluación, de acuerdo a las exigencias impuestas por los elementos de autoaprendizaje que importa la asignación a los alumnos de un rol activo del proceso.

La decisión de adoptar la red Internet como uno de los soportes del proceso de enseñanza del Derecho no fue tomada como una decisión institucional. No ha existido ningún planteamiento al interior de la Facultad en este sentido, sino que más bien surgió como una solución a problemas concretos. El modelo se estructuró para ser aplicado a un diplomado que permitiera llegar a todos los abogados de la región del Maule el cual, por otras razones, no se dictó. Sin embargo, este mismo esquema ya está siendo aplicado en el curso de Derecho procesal III.

\subsection{Materialización del soporte.}

El soporte elegido fue la red Internet a través de un portal alojado en el servidor de la Universidad de Talca. 
(http://www.utalca.cl/Facultad/derecho/diplomado/tutoria/index.html). Previo a la implementación del curso de Derecho procesal III, se ejecutó el Programa piloto de Teoría del Delito con participantes voluntarios, en el cual el soporte (Internet), también se materializó en un sitio web.

\subsection{Recursos.}

Los recursos que se utilizaron en el programa piloto (primera concreción del modelo mediatizado), incluyó la utilización de los siguientes recursos informáticos:

Salón virtual: Un salón de chat en el cual se desarrollaron las sesiones virtuales, primeramente diseñadas de media hora de duración, que luego fueron extendidas a un período que fluctuó entre los cincuenta minutos a una hora.

a) Foro de discusión: Un tablón de anuncios electrónicos en los que los alumnos debatirían sobre temas propuestos por el profesor. Este recurso no tuvo la utilización que se esperaba; su fracaso se debió probablemente a la poca difusión y a la ausencia de 'incentivos' de su utilización.

b) Correo electrónico: El correo electrónico se diseñó como medio de comunicación secundario entre el profesor y los alumnos y entre ellos entre sí. Su labor principal está enfocado a la distribución de informaciones y a la remisión de los actividades semanales que los alumnos debían completar. La utilización de este recurso estuvo dentro de los márgenes esperados. A su vez, en el curso de Derecho procesal III en el que se emplea algunos de estos recursos, el correo electrónico cumple una eficiente labor, ya que es a través de él que los alumnos envían sus trabajos.

c) Libro digital: El programa piloto de teoría del delito contó con un texto guía digital al cual los alumnos tuvieron acceso. Éste se encontraba en formato PDF (Portable Document File) en el sitio web del programa.

\subsection{Metodología.}

La metodología empleada adoptó elementos del autoaprendizaje, pero con una presencia permanente del profesor.

El programa de estudios del curso piloto de teoría del delito, fue diseñado en cinco unidades (introducción, teoría de la conducta, tipicidad, antijuricidad y justificación y culpabilidad) que correspondieron a cinco semanas en total.

En cada semana, los alumnos debían revisar el texto guía, participar en la sesión virtual en la cual debían discutirse los problemas que los propios alumnos propusieran o en un debate generado por el profesor, para luego desarrollar los ejercicios prácticos 
que para cada unidad se elaboraron. Los resultados de estos ejercicios debían ser remitidos por los alumnos al profesor al final de cada semana. En el curso de Derecho procesal III, además, estos trabajos -elaborados sobre la base del programa del cursoson revisados por el profesor y devueltos al alumno con observaciones para su discusión.

Las evaluaciones se diseñaron para ser presenciales y orales.

5.4. Ventajas de la adopción de la informática como herramienta en el proceso de enseñanza y de Internet como su soporte.

La adopción de la informática como herramienta en el proceso de enseñanza del Derecho tiene innegables ventajas, ya que coloca a disposición de los alumnos una serie de recursos de búsqueda, almacenamiento y procesamiento de información que de otra forma se hace más inaccesible, otorga la posibilidad de crear comunidades de estudiantes en función de intereses vocacionales similares y podría significar un ahorro considerable de recursos y un aporte ecológico, como la prescindencia del formato impreso en papel.

Ahora bien, la utilización de la red Internet como soporte -no exclusivo- del proceso de enseñanza del Derecho presenta, además de las ventajas anteriores, la de admitir la materialización -valga la cacofonía- del espíritu universalista de la universidad en el sentido que a través de éste se puede incorporar profesores de otras latitudes ${ }^{14}$ e, incluso, la experiencia de estudiantes de otras universidades.

Pero la realidad de las cosas es que, la utilización de la red Internet como un soporte del proceso considerada aisladamente, puede ser sólo un retoque cosmético del modelo tradicional, que no tiende a superar sus defectos. El empleo de las modernas tecnologías debe venir acompañado de un cambio de switch y de una apuesta decidida por un nuevo modelo que permita el aprovechamiento y contribuya al desarrollo de las capacidades jurídicas en los estudiantes. Se trata, en definitiva, de un nuevo modelo con un nuevo rostro.

Pero así como la inversión en este nuevo rostro deslocalizado puede ser sólo un cambio cosmético, el otro extremo también representa un riesgo, es decir, la despersonalización del proceso de enseñanza. El contacto directo entre el profesor y los alumnos es insustituible y en él radica una parte importante de una buena universidad. Por ello, debe dejarse para el soporte mediatizado, todo aquello que le es endosable (que corresponde a casi la totalidad de lo que se hace en la sala en el modelo

\footnotetext{
${ }^{14}$ Tal como lo contemplaba el proyecto original del diplomado que incluía la participación de un profesor holandós de criminología
} 
tradicional, es decir, el traspaso de la información y conocimientos básicos) y dejar para la sala de clases en esta nueva perspectiva, lo que no puede transmitirse a través de la red: el desarrollo de las habilidades y destrezas que necesita un jurista.

\section{LOS PELIGROS DEL MODELO DESLOCALIZADO.}

Al efectuar la estructuración del modelo deslocalizado también se tuvieron presente los peligros que éste reviste: La transformación de la herramienta del soporte (Internet) en un fin en sí mismo y la discriminación por el acceso a la red.

\subsection{Modelo deslocalizado: Primer peligro.}

En realidad, el primero de los peligros antes anotados no es propiamente del modelo que hemos analizado, sino que más bien dice relación con la definición de la metodología a utilizar. Una correcta opción y una adecuada implementación de una metodología con una fuerte orientación al autoaprendizaje debe evitar el riesgo de transformar el soporte en un fin en sí mismo.

\section{Modelo deslocalizado: Segundo peligro.}

Por último, el segundo de los peligros que pueden constituir un obstáculo al funcionamiento de este modelo deslocalizado, está representado por realidades y decísiones económicas. En efecto, el acceso de todos los alumnos que participen de este modelo está condicionado por el acceso real que, en los tiempos necesarios, tengan a la red y al equipamiento necesario. En la realidad de la Universidad de Talca, éste es sólo un peligro potencial por cuanto existe un relativo libre acceso a terminales de computación conectados a la red Internet. ${ }^{15}$, lo que significa que las posibilidades de

${ }^{15}$ En el Campus Lircay de la Universidad de Talca existen 274 terminales dedicados a uso de los alumnos, mientras que existen 225 puntos de conexión a red disponibles, es decir, existe conexión para un $82,12 \%(225)$ de las estaciones de uso para los alumnos. El acceso a la red Internet es ilimitado durante las veinticuatro horas del día, durante los siete días de la semana. Ello significa que en el Campus Lircay de la Universidad de Talca existe una disponibilidad de 10.080 horas de conexión semanales.

Número de computadores disponibles para académicos, administrativos y alumnos y puntos de red disponibles (Campus Lircay).

\begin{tabular}{|l|c|c|}
\cline { 2 - 3 } \multicolumn{1}{c|}{} & $\begin{array}{c}\text { Computadores } \\
\text { disponibles }\end{array}$ & $\begin{array}{c}\text { Puntos de conexión } \\
\text { a red disponibles }\end{array}$ \\
\hline Académicos & 282 & 355 \\
\hline Administrativos & 222 & 207 \\
\hline Alumnos & 274 & 225 \\
\hline Total & 778 & 787 \\
\hline
\end{tabular}


discriminación en el acceso sean menores, ya que, en total, en la Universidad de Talca hay 13.3 alumnos por cada terminal.

\section{CONCLUSIÓN: EL CAMBIO DEL SOPORTE COMO UN CAMBIO REAL DE MODELO. LA SUSTITUCIÓN DEL CENTRO DE GRAVEDAD DEL PROCESO DEL PROFESOR AL ALUMNO.}

La principal característica de este nuevo modelo que el área de Derecho penal y procesal penal de la Universidad de Talca está tratando de implementar es el cambio del centro de gravedad del proceso: del profesor en el que se encuentra radicado en el modelo tradicional, al alumno en este nuevo modelo; adoptando técnicas propias del autoaprendizaje a través de la entrega gradual y procesada de información.

Las interminables 'bibliografías complementarias' que se acostumbra a acompañar a los programas de estudio, deben ser sustituidas por lecturas precisas de acuerdo a las necesidades del programa. La evaluación acumulativa de un periodo generalmente prolongado, debe ser reemplazada por un instrumento que mida las habilidades a través de la aplicación de la información al desarrollo de hipótesis concretas, en períodos relativamente cortos (una semana o quince días).

Se pretende reducir el número de clases presenciales a un número tal, que permita la interrelación con el profesor y que le conceda al alumno una libertad para administrar el tiempo de acuerdo a sus necesidades e intereses. No se trata de hacer más fácil el proceso de enseñanza, sino que, por el contrario, se pretende reorientar los esfuerzos educativos hacia la formación de licenciados con un fuerte desarrollo de sus aptitudes frente a la vida práctica del desenvolvimiento profesional. 